\title{
Numerical methods in inverse obstacle scattering
}

\author{
Rainer Kress*
}

(Received 7 August 2000)

\begin{abstract}
We consider the inverse problem to determine the shape of an obstacle from a knowledge of the far field pattern for the scattering of time-harmonic acoustic plane waves. This is a model problem for applications in radar, sonar, geophysical exploration, medical imaging and nondestructive testing. It is difficult to solve, since it is nonlinear and extremely ill-posed. Following the historical development over the
\end{abstract}

*Institut für Numerische und Angewandte Mathematik, Universität Göttingen, 37083 Göttingen, GERMANY. mailto:kress@math.uni-goettingen.de

${ }^{0}$ See http: //anziamj . austms .org. au/V42/CTAC99/AKre for this article and ancillary services, (c) Austral. Mathematical Soc. 2000. Published 27 Nov 2000. 
last fifteen years, in this lecture we shall describe the main ideas of three different methods for the approximate numerical solution of the inverse obstacle scattering problem that acknowledge its nonlinearity and ill-posedness.

\section{Contents}

1 Introduction

C46

2 Uniqueness for the inverse problem

C48

3 Ill-posedness of the inverse problem

C49

4 Approximation of the scattered field

C51

5 Regularized Newton iterations

C54

6 Sampling methods

C59

References

C64 


\section{Introduction}

Consider the scattering of a time-harmonic acoustic wave $u^{i}$ by a given impenetrable obstacle described by a bounded domain $D$ in the Euclidean space $\mathbb{R}^{3}$ with a connected boundary $\partial D$. Then the simplest direct obstacle scattering problem is to find the total field $u=u^{i}+u^{s}$ such that $u$ satisfies the Helmholtz equation

$$
\triangle u+k^{2} u=0 \quad \text { in } \mathbb{R}^{3} \backslash \bar{D}
$$

with wave number $k>0$, the Dirichlet boundary condition

$$
u=0 \quad \text { on } \partial D
$$

and the Sommerfeld radiation condition

$$
\lim _{r \rightarrow \infty} r\left(\frac{\partial u^{s}}{\partial r}-i k u^{s}\right)=0, \quad r=|x|,
$$

uniformly with respect to all directions. Physically speaking, the Dirichlet condition (2) corresponds to a sound-soft obstacle $D$. Boundary conditions other than the Dirichlet condition also need to be considered such as the Neumann condition for a sound-hard obstacle or the impedance boundary condition. Furthermore, the scattering by a penetrable obstacle $D$ leads to transmission and resistive boundary conditions. For this lecture the Dirichlet condition is chosen in order to illustrate some basic ideas. Inverse obstacle scattering for acoustic waves is of its own practical interest and simultaneously it serves as a model case for inverse obstacle scattering for electromagnetic and elastic waves. 
Due to the Sommerfeld radiation condition (3) the scattered wave $u^{s}$ has an asymptotic behaviour of the form

$$
u^{s}(x)=\frac{e^{i k|x|}}{|x|}\left\{u_{\infty}\left(\frac{x}{|x|}\right)+O\left(\frac{1}{|x|}\right)\right\}, \quad|x| \rightarrow \infty,
$$

uniformly for all directions. The function $u_{\infty}$, defined on the unit sphere $\Omega$ in $\mathbb{R}^{3}$, is called the far field pattern of the scattered wave. The basic acoustic inverse obstacle scattering problem considered in this lecture is, given the far field pattern $u_{\infty}$ of the scattered wave $u^{s}$ for one or several incident plane waves $u^{i}(x)=e^{i k x \cdot d}$ with incident direction $d \in \Omega$, to determine the shape of the scatterer $D$.

As opposed to the direct scattering problem, which is linear and wellposed in the sense of Hadamard, the inverse problem is nonlinear, since the solution to the direct scattering problem depends nonlinearly on the boundary $\partial D$, and ill-posed, since the construction of the scattered wave $u^{s}$ from its far field pattern $u_{\infty}$ is ill-posed. We want to solve this inverse problem for frequencies in the resonance region, i.e., for scatterers $D$ and wave numbers $k$ such that the wavelengths $2 \pi / k$ is of a comparable size to the diameter of the scatterer. In particular, low frequency methods like impedance tomography or high frequency methods like physical or geometrical optics do not yield valid approximations in the intermediate frequency range. For a detailed study of the inverse obstacle scattering problem we refer to the monograph [4].

Roughly speaking one can distinguish between two different approaches 
for approximately solving the inverse obstacle scattering problem. In one group of methods the inverse obstacle problem is considered as an ill-posed nonlinear operator equation and iterative techniques are employed for its solution. These methods require the solution of the direct scattering problem for different domains at each iteration step. In a second group of methods the need of solving the direct scattering problem is avoided. This is achieved by separating the inverse obstacle scattering problem into a linear ill-posed part for the reconstruction of the scattered wave from the far field pattern and a nonlinear well-posed part for finding the location of the boundary of the scatterer from the boundary condition for the total field. In this lecture we shall outline main ideas of typical methods from both of these two groups.

\section{Uniqueness for the inverse problem}

A first question to ask about the inverse obstacle scattering problem is uniqueness, i.e., the question under what conditions an obstacle is uniquely determined by a knowledge of its far field patterns for incident plane waves. This is of interest both for the theoretical study and the implementation of numerical algorithms. The classical uniqueness result in inverse obstacle scattering is due to Schiffer (see [4]) and stated in the following theorem.

Theorem 1 Assume that $D_{1}$ and $D_{2}$ are two sound-soft scatterers such that the corresponding far field patterns $u_{\infty, 1}=u_{\infty, 2}$ coincide for all observation 
directions $\hat{x} \in \Omega$ and for all incident directions $d \in \Omega$ (for a fixed wave number $k$ ), then $D_{1}=D_{2}$.

We note that a technique suggested by Kirsch and Kress [14] (see also [4], p. 111) for uniqueness proofs in inverse obstacle scattering shows that for the above theorem it is not necessary to know whether the boundary condition for the two obstacles is the Dirichlet, the Neumann or the impedance condition.

Colton and Sleeman [6] (see also [4], p. 107) showed that the scatterer is uniquely determined by a knowledge of the far field pattern for one incident wave provided $D$ is contained in a ball of radius $R$ such that $k R<\pi$. It still remains a challenging open problem to establish if the far field pattern for one incoming plane wave for one single incident direction and one single wave number always determines the scatterer without any a priori information.

\section{Ill-posedness of the inverse problem}

The ill-posedness of the inverse obstacle scattering problem can be illustrated in terms of expansions with respect to spherical harmonics. Assume that $Y_{n}^{m}, n=0,1,2, \ldots, m=-n, \ldots, n$, is an orthonormal basis of spherical harmonics. Then the scattered wave $u^{s}$ as solution to the Helmholtz equation 
satisfying the radiation condition has an expansion of the form

$$
u^{s}(x)=k \sum_{n=0}^{\infty} \sum_{m=-n}^{n} i^{n+1} a_{n}^{m} h_{n}^{(1)}(k|x|) Y_{n}^{m}\left(\frac{x}{|x|}\right)
$$

which is valid for large $|x|$ and where $h_{n}^{(1)}$ denotes the spherical Hankel function of order $n$ and of the first kind. From the asymptotics for the spherical Hankel functions for large $|x|$ it follows that the far field pattern of $u^{s}$ is given by

$$
u_{\infty}=\sum_{n=0}^{\infty} \sum_{m=-n}^{n} a_{n}^{m} Y_{n}^{m} .
$$

Choosing $R>0$ such that $D$ is contained within the sphere of radius $R$ centred at the origin, from Parseval's equality and the asymptotics for the spherical Hankel functions for large $n$ it can be deduced that the coefficients $a_{n}^{m}$ in (5) must satisfy

$$
\sum_{n=0}^{\infty}\left(\frac{2 n}{k e r}\right)^{2 n} \sum_{m=-n}^{n}\left|a_{n}^{m}\right|^{2}<\infty
$$

for all $r>R$. Now consider a perturbation of a given far field pattern $u_{\infty}$ in a norm that is suitable to describe measurement errors, for example in the $L^{2}$ norm. Then, in general, the Fourier coefficients of the perturbed far field pattern $u_{\infty}^{\delta}$ with $\left\|u_{\infty}^{\delta}-u_{\infty}\right\|_{L^{2}(\Omega)} \leq \delta$ for some error level $\delta>0$ will not satisfy the growth condition (7) and therefore no solution to the Helmholtz equation satisfying the radiation condition with far field pattern $u_{\infty}^{\delta}$ will exist, i.e., small perturbations of the given data $u_{\infty}$ will make the problem 
to determine the radiating solution $u^{s}$ to the Helmholtz equation from its far field pattern $u_{\infty}$ unsolvable. Errors in the Fourier coefficient $a_{n}^{m}$ of the far field pattern expansion (6) will be amplified by the factor

$$
h_{n}^{(1)}(k|x|)=O\left(\frac{2 n}{k e r}\right)^{n}
$$

in the series (5) for $u^{s}$. Therefore, small changes in a finite number of the Fourier coefficients of $u_{\infty}$ can cause extremely large changes in $u^{s}$. These large changes in the scattered field $u^{s}$, of course, then will also cause large changes in the location of the boundary determined through $u^{i}+u^{s}=0$ on $\partial D$.

Summarizing, for small changes in the data, in general, no solution to the inverse obstacle scattering problem exists and if it exists it does not depend continuously on the data.

\section{Approximation of the scattered field}

In this section we describe an approximation method for the inverse obstacle scattering problem that was developed by Kirsch and Kress [13]. Its basic idea is to break up the inverse scattering problem into two parts: the first part deals with the ill-posedness by constructing the scattered wave $u^{s}$ from its far field pattern $u_{\infty}$ and the second part deals with the nonlinearity by determining the unknown boundary $\partial D$ of the scatterer as the location of 
the zeros of the total field $u^{i}+u^{s}$. For the first part, we assume a priori that enough information is known about the unknown scattering obstacle $D$ so we can place a closed surface $\Gamma$ inside $D$. We then try to represent the scattered field $u^{s}$ as a single-layer potential

$$
u^{s}(x)=\int_{\Gamma} \varphi(y) \Phi(x, y) d s(y)
$$

with an unknown density $\varphi \in L^{2}(\Gamma)$ in terms of the fundamental solution

$$
\Phi(x, y)=\frac{1}{4 \pi} \frac{e^{i k|x-y|}}{|x-y|}, \quad x \neq y
$$

to the Helmholtz equation. The far field pattern $u_{\infty}$ of the single-layer potential (8) has the representation

$$
u_{\infty}(\hat{x})=\frac{1}{4 \pi} \int_{\Gamma} e^{-i k \hat{x} \cdot y} \varphi(y) d s(y), \quad \hat{x} \in \Omega .
$$

Therefore, if the far field pattern $u_{\infty}$ is given, the density $\varphi$ has to be found by solving the integral equation of the first kind (9). Due to its analytic kernel, the integral equation (9) is severely ill-posed with the singular values of the operator $S_{\infty}: L^{2}(\Gamma) \rightarrow L^{2}(\Omega)$ defined by

$$
\left(S_{\infty} \varphi\right)(\hat{x}):=\frac{1}{4 \pi} \int_{\Gamma} e^{-i k \hat{x} \cdot y} \varphi(y) d s(y), \quad \hat{x} \in \Omega,
$$

decaying at least exponentially. This reflects the ill-posed nature of the problem to determine $u^{s}$ from its far field pattern $u_{\infty}$ as described in the previous 
section. For a stable numerical solution of (9) Tikhonov regularization can be applied, i.e., (9) is replaced by

$$
\alpha \varphi_{\alpha}+S_{\infty}^{*} S_{\infty} \varphi_{\alpha}=S_{\infty}^{*} u_{\infty}
$$

where $S_{\infty}^{*}$ is the $L^{2}$ adjoint of $S_{\infty}$ and $\alpha>0$ denotes a regularization parameter. After having solved (10) for $\varphi_{\alpha}$, the unknown boundary $\partial D$ is then determined by requiring the potential (8) with density $\varphi_{\alpha}$ to satisfy the boundary condition $u^{i}+u^{s}=0$ on $\partial D$. We set

$$
u_{\alpha}(x):=u^{i}(x)+\int_{\Gamma} \varphi_{\alpha}(y) \Phi(x, y) d s(y)
$$

and define an operator

$$
f:\left.\Lambda \rightarrow u_{\alpha}\right|_{\Lambda}
$$

mapping closed surfaces $\Lambda$ containing $\Gamma$ in its interior to the values of $u_{\alpha}$ on $\Lambda$. Then, obviously, we need to solve

$$
f(\Lambda)=0,
$$

what can be done in a least squares sense by minimizing the defect $\|f(\Lambda)\|_{L^{2}(\Lambda)}$ over all surfaces $\Lambda \in V$ contained in some suitable class $V$ of admissible boundary surfaces.

This reformulation of the inverse scattering problem as an optimization problem has been analysed in [13] (see also [4]). For details on its discretization and for three-dimensional examples we refer to the monograph [4] and to Kress and Zinn [18]. 
In closing this section, we also want to mention that, in principle, one can replace the approximation of the scattered field $u^{s}$ through a singlelayer potential by any other convenient approximation. For example, Angell, Kleinman, and Roach [2] suggested using an expansion with respect to radiating spherical wave functions in the sense of (5) instead of the single-layer potential.

Although the methods of Kirsch and Kress and of Angell, Kleinman, and Roach, which were developed in the mid-eighties have been revived through more recent papers (see Angell, Jiang, and Kleinman [1] and Haas and Lehner [8]) they probably will not remain competitive in efficiency with iterative methods such as the regularized Newton method described in the next section. The increase in the computational cost for the Newton method as compared to the methods of Kirsch and Kress and of Angell, Kleinman, and Roach is compensated by notably much more accurate reconstructions. However, the methods of this section will keep their importance as instructive examples for the idea to separate the ill-posedness and the nonlinearity in inverse scattering.

\section{$5 \quad$ Regularized Newton iterations}

Newton's iteration method for solving inverse obstacle scattering problems was first used by Roger [20] in 1981. However, only within the nineties a rigorous foundation was established along the following ideas. 
For a fixed incident field $u^{i}$, the solution to the direct scattering problem defines an operator $\mathcal{F}: \partial D \mapsto u_{\infty}$ that maps the boundary $\partial D$ of the scatterer $D$ onto the far field pattern $u_{\infty}$ of the scattered wave $u^{s}$. In terms of this boundary to far field operator, given a far field pattern $u_{\infty}$, the inverse problem consists in solving the operator equation

$$
\mathcal{F}(\partial D)=u_{\infty}
$$

for the unknown boundary $\partial D$. In order to define the operator $\mathcal{F}$ properly, we choose a fixed reference domain $D$ and consider a family of scatterers $D_{h}$ with boundaries represented in the form

$$
\partial D_{h}=\{x+h(x): x \in \partial D\}
$$

where $h: \partial D \rightarrow \mathbb{R}^{3}$ is twice continuously differentiable and sufficiently small in the $C^{2}$ norm on $\partial D$. Then we may consider the operator $\mathcal{F}$ as a mapping from a sufficiently small ball $V \subset C^{2}(\partial D)$ into $L^{2}(\Omega)$. Note that we can reformulate the uniqueness result of Colton and Sleeman mentioned in Section 2 in terms of injectivity of $\mathcal{F}$. The following theorem on the differentiability of $\mathcal{F}$ with respect to the boundary and the characterization of the derivative is fundamental for the solution of (12) via Newton type iterations.

Theorem 2 The boundary to far field mapping $\mathcal{F}: \partial D_{h} \mapsto u_{\infty}$ is Fréchet differentiable. The derivative is given by

$$
\mathcal{F}^{\prime}(\partial D): h \rightarrow v_{h, \infty},
$$


where $v_{h, \infty}$ is the far field pattern of the solution $v_{h}$ to the Dirichlet problem for the Helmholtz equation in $\mathbb{R}^{3} \backslash \bar{D}$ satisfying the Sommerfeld radiation condition and the boundary condition

$$
v_{h}=-\nu \cdot h \frac{\partial u}{\partial \nu} \quad \text { on } \partial D
$$

Rigorous proofs of this result were first obtained by Kirsch [10], in the sense of a domain derivative via variational methods, and by Potthast [19], in the sense of Fréchet differentiability via boundary integral equation techniques. The boundary condition (14) can be obtained formally by differentiating the boundary condition $u=0$ on $\partial D_{h}$ with respect to $\partial D_{h}$ by the chain rule. We note that the nullspace of the linear operator $\mathcal{F}^{\prime}(\partial D)$ is given by

$$
N\left(\mathcal{F}^{\prime}(\partial D)\right)=\left\{h \in C^{2}(\partial D): \nu \cdot h=0\right\},
$$

i.e., by the tangential fields on $\partial D$. This form of the nullspace reflects the fact that the solution to the scattering problem remains the same when only the parametrization of the boundary $\partial D$ is changed. For computational purposes, this inherent non-uniqueness has to be taken care of by choosing appropriate classes of parametrizations.

Given a far field pattern $u_{\infty}$, the classical Newton method for solving the nonlinear equation (12) consists in replacing it by the linearized equation

$$
\mathcal{F}(\partial D)+\mathcal{F}^{\prime}(\partial D) h=u_{\infty}
$$


that has to be solved for $h$ in order to improve an approximate boundary $\partial D$ into a new approximation $\partial D_{h}$ through (13). In the usual fashion, this procedure is iterated, i.e.,

$$
\mathcal{F}^{\prime}\left(\partial D_{n}\right) h_{n}=u_{\infty}-\mathcal{F}\left(\partial D_{n}\right), \quad n=0,1,2, \ldots,
$$

to obtain a sequence of approximations

$$
\partial D_{n+1}=\left\{x+h_{n}(x): x \in \partial D_{n}\right\} .
$$

Since the operator $\mathcal{F}^{\prime}(\partial D)$ maps $h$ to the far field pattern of the solution to the boundary value problem (14), it is smoothing and therefore compact, i.e., the linear equation (15) inherits the ill-posedness from the nonlinear equation (12). Therefore regularization techniques have to be employed. Using Tikhonov regularization, the classical Newton iteration (16) has to be replaced by

$$
\alpha_{n} h_{n}+\left[\mathcal{F}^{\prime}\left(\partial D_{n}\right)\right]^{*} \mathcal{F}^{\prime}\left(\partial D_{n}\right) h_{n}=\left[\mathcal{F}^{\prime}\left(\partial D_{n}\right)\right]^{*}\left\{u_{\infty}-\mathcal{F}\left(\partial D_{n}\right)\right\}
$$

with a sequence of positive regularization parameters $\alpha_{n}$. Although within the last decade progress has been made in the analysis of regularized Newton methods for solving ill-posed nonlinear operator equations (among others see Deuflhard, Engl, and Scherzer [7]), the convergence of (17) for the inverse obstacle scattering problem at present is not satisfactorily settled with only partial success obtained by Hohage [9].

For practical computations $h$ is taken from a finite dimensional subspace

$$
W_{N}=\operatorname{span}\left\{q_{1}, \ldots, q_{N}\right\} \subset C^{2}(\partial D)
$$


with dimension $N$ and equation (16) is approximately solved by collocating it at $M$ points $\hat{x}_{1}, \ldots, \hat{x}_{M} \in \Omega$. Then writing

$$
h_{n}=\sum_{j=1}^{N} a_{j} q_{j}
$$

one has to solve the linear system

$$
\sum_{j=1}^{N} a_{j}\left(\mathcal{F}^{\prime}\left(\partial D_{n}\right) q_{j}\right)\left(\hat{x}_{i}\right)=u_{\infty}\left(\hat{x}_{i}\right)-\mathcal{F}\left(\partial D_{n}\right)\left(\hat{x}_{i}\right), \quad i=1, \ldots, M,
$$

for the real coefficients $a_{1}, \ldots, a_{N}$. In general, $N \ll M$ and due to the ill-posedness of (15) the overdetermined system (18) is solved by Tikhonov regularization, i.e., by a penalized least squares method. Note that the computational cost of one step of the Newton method is mainly determined by the need to solve the forward scattering problem for the evaluation of the matrix entries $\left(\mathcal{F}^{\prime}\left(\partial D_{n}\right) q_{j}\right)\left(\hat{x}_{i}\right)$ and right-hand sides $\mathcal{F}\left(\partial D_{n}\right)\left(\hat{x}_{i}\right)$ for the approximate boundary $\partial D_{n}$. Note, that in view of the boundary condition (14) computing the cost function $\mathcal{F}\left(\partial D_{n}\right)$ and the derivatives $\mathcal{F}^{\prime}\left(\partial D_{n}\right)\left(q_{j}\right)$ both require the solution of a Dirichlet problem for the Helmholtz equation in the domain bounded by $\partial D_{n}$. Hence, in a boundary integral equation approach for solving the direct obstacle scattering problem, for these computations one needs to solve one boundary integral equation for different right-hand sides, i.e., evaluating the derivative $\mathcal{F}^{\prime}$ does not result in a large increase of the computational costs as compared with the evaluation of $\mathcal{F}$. Nevertheless, the need to solve a direct scattering problem in a domain which changes 
in each iteration step makes the Newton iteration method computationally costly. Note that in a corresponding iteration for the solution of (11) in the method of Section 4 only the evaluation of a potential and its gradient is required.

For details on the implementation in the two-dimensional case and numerical examples we refer to Hohage [9], Kirsch [10, 11] and Kress [15, 16, 17] and for further references to the monograph [4]. Although at the time of this writing no three-dimensional numerical implementations have been reported, the existing numerical examples in two dimensions indicate highly accurate reconstructions and provide evidence for the practicality of regularized Newton methods in inverse obstacle scattering.

\section{Sampling methods}

The methods discussed in the two previous sections and most other methods as described in the monograph [4] rely on some a priori information for obtaining initial approximations to start the corresponding iterative procedures. In this final section we briefly outline the principle ideas of two methods for finding approximations for the solution to the inverse obstacle scattering problem without any use of a priori information on the obstacle. However, these two methods require the knowledge of the far field pattern for all incident and all observation directions whereas the methods of the two previous sections work, in principle, with one incident field. These methods 
have been suggested by Colton and Kirsch [3] and by Kirsch [12] and named sampling methods.

In the sequel, for an incident plane wave $u^{i}(x, d)=e^{i k x \cdot d}$ we will indicate the dependence of the scattered field, of the total field, and of the far field pattern on the incident direction $d$ by writing, respectively, $u^{s}(x, d), u(x, d)$, and $u_{\infty}(\hat{x}, d)$. For $g \in L^{2}(\Omega)$ consider the so-called Herglotz wave function

$$
v^{i}(x):=\int_{\Omega} e^{i k x \cdot d} g(d) d s(d), \quad x \in \mathbb{R}^{3} .
$$

By superposition, the scattered wave corresponding to the incident field $v^{i}$ is given by

$$
v^{s}(x)=\int_{\Omega} u^{s}(x, d) g(d) d s(d), \quad x \in \mathbb{R}^{3} \backslash \bar{D} .
$$

We define the far field operator $F: L^{2}(\Omega) \rightarrow L^{2}(\Omega)$ by

$$
(F g)(\hat{x}):=\int_{\Omega} u_{\infty}(\hat{x}, d) g(d) d s(d), \quad \hat{x} \in \Omega,
$$

and note that $F g$ is the far field pattern of the scattered wave $v^{s}$ corresponding to the Herglotz wave function $v^{i}$ as incident field.

The first sampling method may be considered as a modification of an earlier method proposed by Colton and Monk [5] in the mid-eighties and described in detail in the monograph [4]. Its basic idea is to determine $g$ for the Herglotz wave function $v^{i}$ such that the corresponding scattered wave $v^{s}$ coincides with a point source $\Phi(\cdot, z)$ located at a point $z$ in the interior of 
the scatterer $D$. Denoting the far field pattern of the fundamental solution $\Phi(\cdot, z)$ by

$$
\Phi_{\infty}(\hat{x}, z)=\frac{1}{4 \pi} e^{-i k \hat{x} \cdot z}, \quad \hat{x} \in \Omega,
$$

obviously this requires to find $g=g(\cdot, z)$ as a solution to the integral equation of the first kind

$$
F g(\cdot, z)=\Phi_{\infty}(\cdot, z) .
$$

If $g$ solves equation (19) we have $v^{s}=\Phi(\cdot, z)$ and in view of the boundary condition

$$
v^{i}+\Phi(\cdot, z)=0 \quad \text { on } \partial D .
$$

we conclude that

$$
\|g(\cdot, z)\|_{L^{2}(\Omega)} \rightarrow \infty
$$

as the source point $z$ approaches the boundary $\partial D$. Therefore, in principle, the boundary $\partial D$ may be found by solving the integral equation (19) for $z$ taken from a sufficiently fine grid in $\mathbb{R}^{3}$ and determining $\partial D$ as the location of those points $z$ where $\|g(\cdot, z)\|_{L^{2}(\Omega)}$ becomes large.

However, unfortunately, the integral equation of the first kind (19) can be shown to have a solution only in special cases (for example if $D$ is a ball with centre at $z$ ). Nevertheless, the following result can be established (see Colton and Kirsch [3]).

Theorem 3 Assume that the Dirichlet problem for the Helmholtz equation in $D$ with homogeneous boundary condition has only the trivial solution, then 
for every $\varepsilon>0$ and $z \in D$ there exists a function $g(\cdot, z) \in L^{2}(\Omega)$ such that

$$
\left\|F g(\cdot, z)-\Phi_{\infty}(\cdot, z)\right\|_{L^{2}(\Omega)} \leq \varepsilon
$$

and

$$
\|g(\cdot, z)\|_{L^{2}(\Omega)} \rightarrow \infty, \quad z \rightarrow \partial D
$$

From this it can be expected that solving the integral equation (19) and scanning the values for $\|g(\cdot, z)\|_{L^{2}(\Omega)}$ will yield an approximation for $\partial D$ through those points where the norm of $g$ is large. A possible procedure with noisy data

$$
\left\|u_{\infty, \delta}-u_{\infty}\right\|_{L^{2}(\Omega \times \Omega)} \leq \delta
$$

with error level $\delta$ is as follows. Denote by $F_{\delta}$ the far field operator $F$ with the kernel $u_{\infty}$ replaced by the data $u_{\infty, \delta}$. Then for each $z$ from a grid in $\mathbb{R}^{3}$ determine $g^{\delta}=g^{\delta}(\cdot, z)$ by minimizing the Tikhonov functional

$$
\left\|F_{\delta} g^{\delta}(\cdot, z)-\Phi_{\infty}(\cdot, z)\right\|_{L^{2}(\Omega)}^{2}+\alpha\left\|g^{\delta}(\cdot, z)\right\|_{L^{2}(\Omega)}^{2},
$$

where the regularization parameter $\alpha$ is chosen according to Morozov's discrepancy principle, i.e., $\alpha=\alpha(z)$ is chosen such that

$$
\left\|F_{\delta} g^{\delta}(\cdot, z)-\Phi_{\infty}(\cdot, z)\right\|_{L^{2}(\Omega)} \approx \delta
$$

Then the unknown boundary is determined by those points where the norm $\left\|g^{\delta}(\cdot, z)\right\|_{L^{2}(\Omega)}$ increases. For numerical examples in two dimensions we refer to Colton and Kirsch [3]. 
In addition to the fact that the integral equation (19), in general, is not solvable, the above Theorem 3 does not say anything about $\|g(\cdot, z)\|_{L^{2}(\Omega)}$ for $z \in \mathbb{R}^{3} \backslash \bar{D}$. These drawbacks of the first sampling method were remedied by Kirsch [12] who, in a second sampling method, suggested to replace (19) by

$$
\left(F^{*} F\right)^{1 / 4} g(\cdot, z)=\Phi_{\infty}(\cdot, z)
$$

Theorem 4 As in Theorem 3 assume that the Dirichlet problem for the Helmholtz equation in $D$ with homogeneous boundary condition has only the trivial solution, then $z \in D$ if and only if (20) has a solution $g \in L^{2}(\Omega)$.

This explicit characterization of the scatterer in terms of the solvability of the equation of the first kind (20) can be used for a reconstruction with the aid of a singular system $\left(\mu_{n}, \psi_{n}, g_{n}\right)$ of the operator $F$. Then, in terms of this singular system, by Picard's theorem we have that $z \in D$ if and only if

$$
\sum_{n=1}^{\infty} \frac{\left|\left(\psi_{n}, \Phi_{\infty}(\cdot, z)\right)\right|^{2}}{\mu_{n}}<\infty,
$$

where $(\cdot, \cdot)$ denotes the inner product on $L^{2}(\Omega)$.

At a first glance Theorem 4 seems to imply that the nonlinear inverse problem has been completely solved through a linear problem. However, there is still a nonlinear problem involved for finding those point $z$ where (21) is satisfied. Of course, analogous to the sampling method using (19), an 
obvious way to approximately solve this nonlinear problem is by truncating the series (21) through a finite sum for $z$ on a grid in $\mathbb{R}^{3}$ and determine $\partial D$ as the location of those points $z$ where this sum becomes large. For the numerical implementation and examples in two dimensions we refer to Kirsch [12].

Both sampling methods described in this section can be viewed as methods breaking up the inverse obstacle scattering problem into a linear ill-posed problem and a nonlinear well-posed problem. However, as opposed to the methods of Section 4 the sampling methods have the following advantages: they do not need any a priori information on the scattering obstacle, they also work for the Neumann boundary condition without the need to know the type of the boundary condition, and they work for scatterers having more than one component. However, on the other hand, the sampling methods require knowledge of the far field pattern for all incident and all observation directions.

In general, the sampling methods will not lead to very sharply defined boundaries. However, if necessary these approximate boundaries then could be improved for example by using the regularized Newton iteration from the previous section.

\section{References}


[1] T.S. Angell, X. Jiang, and R.E. Kleinman. On a numerical method for inverse acoustic scattering. Inverse Problems, 13:531-545, 1997. C54

[2] T.S. Angell, R.E. Kleinman, and G.F. Roach. An inverse transmission problem for the Helmholtz equation. Inverse Problems, 3:149-180, 1987. C54

[3] D. Colton and A. Kirsch. A simple method for solving inverse scattering problems in the resonance region. Inverse Problems, 12:383-393, 1996. C60, C61, C62

[4] D. Colton and R. Kress. Inverse Acoustic and Electromagnetic Scattering Theory. 2nd. ed. Springer-Verlag, Berlin, 1998. C47, C48, C49, C49, C53, C53, C59, C59, C60

[5] D. Colton and P. Monk. A novel method for solving the inverse scattering problem for time-harmonic acoustic waves in the resonance region. SIAM J. Appl. Math., 45:1039-1053, 1985. C60

[6] D. Colton and B.D. Sleeman. Uniqueness theorems for the inverse problem of acoustic scattering. IMA J. Appl. Math., 31:253-259, 1983. C49

[7] P. Deuflhard, H.W. Engl, and O. Scherzer. A convergence analysis of iterative methods for the solution of nonlinear ill-posed problems under affinely invariant conditions. Inverse Problems, 14:1081-1106, 1998. 
[8] M. Haas and G. Lehner. Inverse 2D obstacle scattering by adaptive iteration. IEEE Transactions on Magnetics, 33:1958-1961, 1997. C54

[9] T. Hohage. Convergence rates of a regularized Newton method in sound-hard inverse scattering. SIAM J. Numer. Anal., 36:125-142, 1998. C57, C59

[10] A. Kirsch. The domain derivative and two applications in inverse scattering theory. Inverse Problems, 9:81-96, 1993. C56, C59

[11] A. Kirsch. Numerical algorithms in inverse scattering theory. In Jarvis and Sleeman, editors, Ordinary and Partial Differential Equations, Vol. IV, , pages 93-111, London, 1993. Pitman Research Notes in Mathematics 289, Longman. C59

[12] A. Kirsch. Characterization of the shape of the scattering obstacle by the spectral data of the far field operator. Inverse Problems, 14:1489-1512, 1998. C60, C63, C64

[13] A. Kirsch and R. Kress. An optimization method in inverse acoustic scattering. In Brebbia et al, editors, Boundary elements IX, Vol 3. Fluid Flow and Potential Applications, pages 3-18, Berlin Heidelberg New York, 1987. Springer-Verlag. C51, C53

[14] A. Kirsch and R. Kress. Uniqueness in inverse obstacle scattering. Inverse Problems, 9:285-299, 1993. C49 
[15] R. Kress. A Newton method in inverse obstacle scattering. In Bui et al, editors, Inverse Problems in Engineering Mechanics, pages 425-432, Balkema, Rotterdam, 1994. C59

[16] R. Kress. Integral equation methods in inverse obstacle scattering. Engineering Anal. with Boundary Elements, 15:171-179, 1995. C59

[17] R. Kress. Integral equation methods in inverse acoustic and electromagnetic scattering. In Ingham and Wrobel, editors, Boundary Integral Formulations for Inverse Analysis, pages 67-92, Southampton, 1997. Computational Mechanics Publications. C59

[18] R. Kress and A. Zinn. On the numerical solution of the three dimensional inverse obstacle scattering problem. J. Comp. Appl. Math., 42:49-61, 1992. C53

[19] R. Potthast. Fréchet differentiability of boundary integral operators in inverse acoustic scattering. Inverse Problems, 10:431-447, 1994. C56

[20] A. Roger. Newton Kantorovich algorithm applied to an electromagnetic inverse problem. IEEE Trans. Ant. Prop., AP-29:232-238, 1981. C54 